\title{
Many Types of Stability of Abstract First and Second Order Linear Dynamic Equations on Time Scales
}

\author{
Alaa E. Hamza, Gamal A. F. Ismail*, Dina Ahmed* \\ Department of Mathematics, Faculty of science, University of Jeddah, Jeddah, 21589, \\ Saudi Arabia \\ Cairo University, Department of Mathematics, Giza, Egypt \\ E-mail: hamzaaeg2003@yahoo.com \\ *Department of Mathematics, Faculty of Girls, Ain Shams University, Cairo, Egypt \\ E-mail: gam ismail@yahoo.com and dina.ahmed234@yahoo.com
}

\begin{abstract}
In this paper we investigate sufficient conditions for many types of stability of both of the abstract first order linear dynamic equations on time scales of the form

$$
x^{\Delta}(t)+A(t) x(t)=\mathrm{f}(\mathrm{t}), \mathrm{t} \in \mathbb{T},
$$

and the second order linear dynamic equations of the form

$$
x^{\Delta \Delta}(t)+A(t) x^{\Delta}(t)+R(t) x(t)=f(t), t \in \mathbb{T},
$$

Where $A, R: \mathbb{T} \rightarrow L(\mathbb{X})$, the space of all bounded linear operators from a Banachspace $\mathbb{X}$ into itself, and $f$ is rd-continuous from a time scale $\mathbb{T}$ to $\mathbb{X}$. Some givenillustrative examples show the applicability of the main results.
\end{abstract}

Mathematics Subject Classifications:26E70, 34N05, 34K20, 39A30.

Keywords:Time scales, Linear dynamic equations, Stability theory.

\section{Preliminaries and introduction}

The objective of the theory of dynamic equations on time scales is to unify continuous and discrete calculus $[3,15]$ which was introduced by Stefan Hilger [14]. For more detailes about this theory, we refer the reader to the very interesting monographs [4] and [5]. In recent years, there has been an increasing interest in studying the asymptotic behavior of solutions of dynamic equations on time scales due to its applications in many fields especially in biology, economics. In [10] DUC, Ilchmann, Siegmund and Taraba derived sufficient conditions for stability and asymptotic stability of linear time varying second order scalar differential equations of the form:

$$
\ddot{x}+a_{1}(t) \dot{x}+a_{0}(t) x=0 .
$$

Drozdowicz and Popenda, in [9], investigated the asymptotic behavior of solutions of second order difference equations. We refer the reader to the monograph [11].

In [12], Hamza and Oraby studied many types of stability of solutions of the first order linear dynamic equation of the form: 


$$
\left\{\begin{array}{c}
u^{\Delta}=A u(t), \quad t \in \mathbb{T}, \quad t>0 \\
u(0)=x \in \mathbb{X}
\end{array}\right.
$$

where $A$ is the generator of a $C_{0}$ - semigroup $\{T(t): t \in \mathbb{T}\}$; the space of all bounded linear operators from a Banach space $X$ into itself. Here $\mathbb{T} \in\left\{\mathbb{Z}^{\geq 0}, \mathbb{R}^{\geq 0}\right\}$. For related results, see [1, 6-10, 17].

In this paper, we obtain some new results concerning with many types of stability like (exponential stability, uniform exponential stability, $h$-stability and uniform $h-$ stability) of abstract first order linear dynamic equations of the form:

$$
x^{\Delta}(t)+A(t) x(t)=\mathrm{f}(\mathrm{t}), \mathrm{t} \in \mathbb{T} .
$$

We use these results to establish sufficient conditions for the stability of the abstract second order dynamic equations of the form

$$
x^{\Delta \Delta}(t)+A(t) x^{\Delta}(t)+R(t) x(t)=f(t), t \in \mathbb{T},
$$

where $A, R: \mathbb{T} \rightarrow L(\mathbb{X})$, and $f$ is rd-continuous from a time scale $\mathbb{T}$ to a Banach space $\mathbb{X}$. Finally, we give some illustrative examples to show the applicability of the theoritical results.

Now we exihibite the concepts of stability, uniform stability, exponential stability, uniform exponential stability, $h$-stability and uniform $h$-stability, of the general dynamic equations of the form

$$
x^{\Delta^{n}}(t)=F\left(t, x(t), x^{\Delta}(t), \ldots, x^{\Delta^{n-1}}(t)\right), t \geq \tau, t, \tau \in \mathbb{T},
$$

where $F: \mathbb{T} \times \mathbb{X}^{n} \rightarrow \mathbb{X i s}$ rd-continuous in $\mathrm{t}$ with $F(t, 0, \ldots, 0)=0 ; t \in \mathbb{T}$. These concepts include the boundedness of solutions. See $[2,16,18]$. We denote by $x(t)=$ $x\left(t, \tau, x_{\tau}^{0}, \ldots x_{\tau}^{n-1}\right)$ for the solution corresponding to the initial values $x^{\Delta^{i}}(\tau)=x_{\tau}^{i} \in X, i=0, \ldots, n-1$ and we denote by $X(\tau)=\left(x_{\tau}^{0}, \ldots, x_{\tau}^{n-1}\right) \in \mathbb{X}^{n}$ for the $n$-tuple composed of the initial values. We call $X(\tau)$ is an initial state. Assume that $X$ is endowed with a norm $\|$.$\| and \mathbb{X}^{n}$ is the Banach space endowed with the norm $\left\|x_{1}, \ldots, x_{n}\right\|=\sum_{i=1}^{n}\left\|x_{i}\right\|$.

Definition 1.1.Eq.(1.1) is called stable if for every $\tau \in \mathbb{T}$ and for every $\epsilon>0$ there exists $\delta=\delta(\epsilon, \tau)>0$ such that for any two solutions $x(t)=x\left(t, \tau, x_{\tau}^{0}, \ldots, x_{\tau}^{n-1}\right)$ and $\bar{x}(t)=$ $\bar{x}\left(t, \tau, \bar{x}_{\tau}^{0}, \ldots, \bar{x}_{\tau}^{n-1}\right)$ of Eq.(1.1), corresponding to the initial states $X(\tau)=\left(x_{\tau}^{0}, \ldots, x_{\tau}^{n-1}\right)$ and $\bar{X}(\tau)=\left(\bar{x}_{\tau}^{0}, \ldots, \bar{x}_{\tau}^{n-1}\right)$ respectively, we have

$$
\|X(\tau)-\bar{X}(\tau)\|<\delta \Rightarrow\|x(t)-\bar{x}(t)\|<\epsilon, t \geq \tau, t \in \mathbb{T} .
$$

Definition 1.2. Eq.(1.1) is called uniformly stable if for every $\epsilon>0$ there exists $\delta=\delta(\epsilon)>$ 0 such thatfor every $\tau \in \mathbb{T}$ and for any two solutions $x(t)=x\left(t, \tau, x_{\tau}^{0}, \ldots, x_{\tau}^{n-1}\right)$ and $\bar{x}(t)=\bar{x}\left(t, \tau, \bar{x}_{\tau}^{0}, \ldots, \bar{x}_{\tau}^{n-1}\right)$ of Eq.(1.1), corresponding to the initial states $X(\tau)$ and $\bar{X}(\tau)$, we have

$$
\|X(\tau)-\bar{X}(\tau)\|<\delta \Rightarrow\|x(t)-\bar{x}(t)\|<\epsilon, t \geq \tau, t \in \mathbb{T}
$$


Definition 1.3.Eq.(1.1) is called exponentially stable if there exists a constant $\alpha>0$ with $-\alpha \in \mathcal{R}^{+}$such that for every $\tau \in \mathbb{T}$, there is $\gamma \in C_{r d}\left(\mathbb{T} \times \mathbb{R}^{+}, \mathbb{R}^{\geq 1}\right)$ such that, anysolution $x(t)=x\left(t, \tau, x_{\tau}^{0}, \ldots, x_{\tau}^{n-1}\right)$ corresponding to the initial state $X(\tau)$ of Eq.(1.1), satisfies

$$
\|x(t)\| \leq \gamma(\tau,\|X(\tau)\|) e_{-}(-\alpha)(t, \tau), t \geq \tau, t \in \mathbb{T} .
$$

Here $\mathcal{R}^{+}$is the family of all positively regressive functions [4].

Definition 1.4. Eq.(1.1) is called uniformly exponentially stable if $\gamma$ is independent on $\tau \in \mathbb{T}$.

Definition 1.5. Let $h: \mathbb{T} \rightarrow \mathbb{R}$ be a positive bounded function. We say that Eq.(1.1) is called $h$-stable if there exists $\gamma \in C_{r d}\left(\mathbb{T} \times \mathbb{R}^{+}, \mathbb{R}^{\geq 1}\right)$ such that for any solution $x\left(t, \tau, x_{\tau}^{0}, \ldots, x_{\tau}^{n-1}\right)$ corresponding to the initial state $X(\tau)$ of Eq.(1.1), we have

$$
\|x(t)\| \leq \gamma(\tau,\|X(\tau)\|) h(t) h(\tau)^{-1}, t \geq \tau, t \in \mathbb{T} .
$$

(Here $\left.h(\tau)^{-1}=\frac{1}{h(\tau)}\right)$.

Definition 1.6. Eq.(1.1) is called uniformly $h-$ stable if $\gamma$ is independent on $\tau \in \mathbb{T}$.

The initial value problem

$$
C P(0): \quad x^{\Delta}(t)=A(t) x(t), \quad x(\tau)=x_{\tau}^{0} \in \mathbb{X}, t \geq \tau, t \in \mathbb{T},
$$

has the unique solution

$$
x(t)=e_{A}(t, \tau) x_{\tau}^{0},
$$

And the initial value problem

$$
C P(f): \quad x^{\Delta}(t)=A(t) x(t)+f(t), \quad x(\tau)=x_{\tau}^{0} \in \mathbb{X}, t \geq \tau, t \in \mathbb{T},
$$

Has the unique solution

$$
x(t)=e_{A}(t, \tau) x_{\tau}^{0}+\int_{\tau}^{t} e_{A}(t, \sigma(s)) f(s) \Delta s,
$$

where $A \in C_{r d}(\mathbb{T}, L(\mathbb{X}))$ and $f \in C_{r d}(\mathbb{T}, \mathbb{X})$. The formula (1.3) is called the variation of parameters formula. Here, $e_{A}(t, \tau)$ is the exponential abstract operator function. For properties of $e_{A}(t, s), t \geq s, t, s \in \mathbb{T}$ see [13]. For instance, it satisfies $e_{A}(t, s)+e_{A}(s, \tau)=$ $e_{A}(t, \tau), t, s, \tau \in \mathbb{T}$ which is called the semigroup property and $e_{A}(t, t) \equiv I$. By linearity of $C P(0)$, we get the equivalence between the stability of $C P(0)$ and the stability of $C P(f)$. We introduce the notions of many types of stability of the family $\left\{e_{A}(t, \tau): t, \tau \in\right.$ $\mathbb{T}, t \geq \tau\}$.

Definition 1.7. We say that family $\left\{e_{A}(t, \tau): t, \tau \in \mathbb{T}, t \geq \tau\right\}$ is

i. stable if there is $\gamma(\tau) \in \mathbb{R}^{+}$such that

$$
\left\|e_{A}(t, \tau)\right\| \leq \gamma(\tau), t \geq \tau, \quad t \in \mathbb{T} .
$$


ii. uniformly stable if there is a positive constant number $\gamma$ such that

$$
\left\|e_{A}(t, \tau)\right\| \leq \gamma, t \geq \tau, \quad t, \tau \in \mathbb{T} .
$$

iii. exponentially stable if there are $\alpha>0$ with $-\alpha \in \mathcal{R}^{+}$and $\gamma \in C_{r d}\left(\mathbb{T} \times \mathbb{R}^{+}, \mathbb{R}^{\geq 1}\right)$ such that

$$
\left\|e_{A}(t, \tau)\right\| \leq \gamma(\tau) e_{-\alpha}(t, \tau), t \geq \tau, \quad t \in \mathbb{T} .
$$

In this case, it is called exponentially stable of type $\alpha, \gamma(\tau)$.

iv. uniformly exponentially stable if there are $\alpha>0$ with $-\alpha \in \mathcal{R}^{+}$and $\gamma \in \mathbb{R}^{\geq 1}$ such that

$$
\left\|e_{A}(t, \tau)\right\| \leq \gamma(\tau) e_{-\alpha}(t, \tau), t \geq \tau, \quad t, \tau \in \mathbb{T} .
$$

In this case, it is called uniformly exponentially stable of type $\alpha, \gamma$.

v. $\quad h$-stable if there is $\gamma \in C_{r d}\left(\mathbb{T}, \mathbb{R}^{\geq 1}\right)$ such that

$$
\left\|e_{A}(t, \tau)\right\| \leq \gamma(\tau) h(t) h(\tau)^{-1}, t \geq \tau, \quad t \in \mathbb{T} .
$$

In this case, it is called $h$-stable of type $\gamma(\tau)$.

vi. uniformly $h$-stable if there is $\gamma \in \mathbb{R}^{\geq 1}$ such that

$$
\left\|e_{A}(t, \tau)\right\| \leq \gamma(\tau) h(t) h(\tau)^{-1}, t \geq \tau, \quad t, \tau \in \mathbb{T} .
$$

In this case, it is called uniformlyh -stable of type $\gamma$.

We need the following two results from [1] concerning the stability of $C P(0)$ and $C P(f)$.

Theorem 1.8. The following conditions are equivalent

i. $\quad C P(0)$ is stable.

ii. $\quad C P(f)$ is stable.

iii. $\quad\left\{e_{A}(t, \tau): t, \tau \in \mathbb{T}, t \geq \tau\right\}$ is stable.

Theorem 1.9. The following conditions are equivalent

i. $\quad C P(0)$ is uniformly stable.

ii. $\quad C P(f)$ is uniformly stable.

iii. $\quad\left\{e_{A}(t, \tau): t, \tau \in \mathbb{T}, t \geq \tau\right\}$ is uniformly stable

\section{Exponential stability ( $h$-stability) of $C P(f)$}

This section is devoted to studying the exponential stability ( $h$-stability) of the nonhomogeneous abstract Cauchy problem $C P(f)$ in terms of the exponential stability 
(h-stability) of the family $\left\{e_{A}(t, \tau): t, \tau \in \mathbb{T}, t \geq \tau\right\}$.

Theorem 2.1. If the following conditions

i. $\left\{e_{A}(t, \tau): t, \tau \in \mathbb{T}, t \geq \tau\right\}$ is exponential stable, with type $\alpha, \gamma(\tau)$,

ii. there exists $\beta=\beta(\tau) \geq 0$ such that $\int_{\tau}^{t} \frac{\|f(s)\| \gamma(\sigma(s))}{1-\mu(s) \alpha} e_{-\alpha}(\tau, s) \Delta s \leq \beta, t \in \mathbb{T}$,

are satisfied, then $C P(f)$ is exponentially stable and every solution $x$ with initial value $x_{\tau}^{0}$ satisfies the following inequality

$$
\|x(t)\| \leq\left(\gamma(\tau)\left\|x_{\tau}^{0}\right\|+\beta\right) e_{-\alpha}(t, \tau) .
$$

Proof. Let $x(t)$ be a solution of $C P(f)$ with initial value $x_{\tau}^{0}$. Then using formula (1.3), we obtain

$$
\|x(t)\| \leq \gamma e_{-\alpha}(t, \tau)\left\|x_{\tau}^{0}\right\|+\int_{\tau}^{t}\|f(s)\| \gamma(\sigma(s)) e_{-\alpha}(t, \sigma(s)) \Delta s
$$

$\leq\left(\gamma\left\|x_{\tau}^{0}\right\|+\beta\right) e_{-\alpha}(t, \tau)$.

There, $C P(f)$ is exponentially stable.

Theorem 2.2. If the following conditions

i. $\left\{e_{A}(t, \tau): t, \tau \in \mathbb{T}, t \geq \tau\right\}$ is uniformly exponential stable, with type $\alpha, \gamma$,

ii. there exists $\beta \geq 0$ (independent of $\tau$ ) such that $\int_{\tau}^{t} \frac{\|f(s)\|}{1-\mu(s) \alpha} e_{-\alpha}(\tau, s) \Delta s \leq \beta, t, \tau \in$ $\mathbb{T}$,

are satisfied, then $C P(f)$ is uniformly exponentially stable.

Proof. The proof is similar to the proof of Theorem 2.1 and will be omitted.

Theorem 2.3. If the following conditions

i. $\left\{e_{A}(t, \tau): t, \tau \in \mathbb{T}, t \geq \tau\right\}$ is $h$-stable, with type $\gamma$,

ii. there exists $\beta=\beta(\tau) \geq 0$ such that $\int_{\tau}^{t} \frac{\|f(s)\| \gamma(\sigma(s))}{h(\sigma(s)) h(\tau)^{-1}} \Delta s \leq \beta, t \in \mathbb{T}$,

are satisfied, then $C P(f)$ is $h$-stable and every solution $x$ with initial value $x_{\tau}^{0}$ satisfies the following inequality

$$
\|x(t)\| \leq\left(\gamma(\tau)\left\|x_{\tau}^{0}\right\|+\beta\right) h(t) h(\tau)^{-1} .
$$

Proof. Let $x(t)$ be a solution of $C P(f)$ with initial value $x_{\tau}^{0}$. Then it satisfies

$$
\|x(t)\| \leq \gamma\left\|x_{\tau}^{0}\right\| h(t) h(\tau)^{-1}+\beta h(t) h(\tau)^{-1}
$$


$\leq\left(\gamma\left\|x_{\tau}^{0}\right\|+\beta\right) h(t) h(\tau)^{-1} \forall t \geq \tau, t \in \mathbb{T}$.

Therefore, $C P(f)$ is $h$-stable.

Theorem 2.4. If the following conditions

i. $\left\{e_{A}(t, \tau): t, \tau \in \mathbb{T}, t \geq \tau\right\}$ is uniformly $h$-stable, with type $\gamma$,

ii. there exists $\beta \geq 0$ (independent of $\tau$ ) such that $\int_{\tau}^{t} \frac{\|f(s)\|}{h(\sigma(s)) h(\tau)^{-1}} \Delta s \leq \beta$,

are satisfied, then $C P(f)$ is uniformly $h$-stable.

Proof. The proof is similar to the proof of Theorem 2.3 and will be omitted.

Theorems 2.3 and 2.4 yield Theorems 2.1 and 2.2 respectively, by putting $h(t) h(\tau)^{-1}=$ $e_{-\alpha}(t, \tau)$.

\section{Stability of second order linear dynamic equations on time scales}

section we establish many types of stability of the second order non-homogeneous dynamic equations of the form

$$
x^{\Delta \Delta}(t)+A(t) x^{\Delta}(t)+R(t) x(t)=f(t), t>\tau, t \in \mathbb{T},
$$

with initial conditions $x^{\Delta^{i}}(\tau)=x_{\tau}^{i} \in \mathbb{X}, i=0,1$ where $A, R: \mathbb{T} \rightarrow L(\mathbb{X})$ and $f \in C_{r d}(\mathbb{T}, \mathbb{X})$. Let $z: \mathbb{T} \rightarrow L(\mathbb{X})$ be a particular solution of the corresponding Riccati equation

$$
z^{\Delta}(t)+\left(A(t)-z^{\sigma}(t)\right) z(t)=R(t), t \in \mathbb{T} .
$$

Assume that $D=z^{\sigma}-A$, -z are regressive and $D \in C_{r d}(\mathbb{T}, L(\mathbb{X}))$.

We need the following lemma. It proof is straightforward and will be omitted.

Lemma 3.1. If $\mathrm{x}$ is a solution of Eq.(3.1), then $g(t)=x^{\Delta}(t)+z(t) x(t)$ is a solution of

$$
g^{\Delta}-D g-f=0
$$

Theorem 3.2.If the functions $\left\|e_{D}(t, \tau)\right\|,\left\|e_{-z}(t, \tau)\right\|$ and $\int_{\tau}^{t}\left\|e_{-z}(t, \sigma(s))\right\| \Delta s$ are bounded for every $\tau \in \mathbb{T}$, then Eq.(3.1) is stable.

Proof.We denote by $K=\sup _{t \geq \tau} \int_{\tau}^{t}\left\|e_{-z}(t, \sigma(s))\right\| \Delta s, L=\sup _{t \geq \tau}\left\|e_{-z}(t, \tau)\right\|$ and $M=\sup _{t \geq \tau}\left\|e_{D}(t, \tau)\right\|$. The equation $g^{\Delta}-D g-f=0$ is stable by Theorem 1.8, since $\left\{e_{D}(t, \tau): t \geq \tau\right\}$ is stable. Let $\epsilon>0$. There is $\delta_{1}(\epsilon, \tau)>0$ such that forany two solutions $g(t)=g\left(t, \tau, g_{\tau}\right)$ and $\bar{g}(t)=\bar{g}\left(t, \tau, \bar{g}_{\tau}\right)$ with initial values $g_{\tau}$ and $\bar{g}_{\tau}$ respectively, we have

$$
\left\|g_{\tau}-\bar{g}_{\tau}\right\|<\delta_{1} \Rightarrow\|g(t)-\bar{g}(t)\|<\frac{\epsilon}{2 K}
$$

Choose $\delta>0$ such that

$$
\delta \leq \min \left(\frac{\delta_{1}}{\max (\|z(\tau)\|, 1)}, \frac{\epsilon}{2 L}\right)
$$


Let $x(t)=x\left(t, \tau, x_{\tau}^{0}, x_{\tau}^{1}\right)$ and $\bar{x}(t)=\bar{x}\left(t, \tau, \bar{x}_{\tau}^{0}, \bar{x}_{\tau}^{1}\right)$ be two solutions with initial states $X(\tau)=\left(x_{\tau}^{0}, x_{\tau}^{1}\right)$ and $\bar{X}(\tau)=\left(\bar{x}_{\tau}^{0}, \bar{x}_{\tau}^{1}\right)$ such that

$$
\|X(\tau)-\bar{X}(\tau)\|<\delta
$$

Hence, $g(t)=x^{\Delta}(t)+z(t) x(t)$ and $\bar{g}(t)=\bar{x}^{\Delta}(t)+z(t) \bar{x}(t)$ are solutions of Eq.(3.3)corresponding to the initial conditions

$$
g_{\tau}=x^{\Delta}(\tau)+z(\tau) x(\tau) \text { and } \bar{g}_{\tau}=\bar{x}^{\Delta}(\tau)+z(\tau) \bar{x}(\tau) ; \text { respectively. }
$$

We see that $\left\|g_{\tau}-\bar{g}_{\tau}\right\|<\delta_{1}$. Consequently, $\|g(t)-\bar{g}(t)\|<\frac{\epsilon}{2 K}, \forall t \geq \tau, t \in \mathbb{T}$. Thesolutions $x(t)$ and $\bar{x}(t)$ of Eq.(3.1) are given by

And

$$
x(t)=e_{-z}(t, \tau) x_{\tau}^{0}+\int_{\tau}^{t} e_{-z}(t, \sigma(s)) g(s) \Delta s
$$

$$
\bar{x}(t)=e_{-z}(t, \tau) \bar{x}_{\tau}^{0}+\int_{\tau}^{t} e_{-z}(t, \sigma(s)) \bar{g}(s) \Delta s
$$

This implies that $\|x(t)-\bar{x}(t)\|<\epsilon$. Therefore, Eq.(3.1) is stable.

Theorem 3.3. If the function $\|z(t)\|$ is bounded and the functions $\left\|e_{D}(t, \tau)\right\|,\left\|e_{-z}(t, \tau)\right\|$ and $\int_{\tau}^{t}\left\|e_{-z}(t, \sigma(s))\right\| \Delta s$ are uniformly bounded with respect $\tau \in \mathbb{T}$, then Eq.(3.1) is uniformly stable.

Proof. The proof is very similar to the proof of Theorem 3.2 and will be omitted.

Theorem 3.4. Assume that the following conditions

i. There exist constants $\alpha>0$ and $\alpha_{1}>0$, and there is $\gamma \in C_{r d}\left(\mathbb{T}, \mathbb{R}^{\geq 1}\right)$ such that $\left\{e_{D}(t, \tau): t \geq \tau\right\}$ (resp. $\left.\left\{e_{-z}(t, \tau): t \geq \tau\right\}\right)$ is stable, with type $\alpha$ and $\gamma(\tau)$ (resp. with type $\alpha_{1}$ and $\left.\gamma(\tau)\right)$,

ii. There are constants $\beta>0$ and $l>0$, dependent on $\tau$, such that

$$
\int_{\tau}^{t} \frac{\|f(s)\| \gamma(\sigma(s))}{1-\mu(s) \alpha} e_{-z}(\tau, s) \Delta s \leq \beta, \quad t \geq \tau
$$

and

$$
\int_{\tau}^{t} \frac{\gamma(\sigma(s))}{1-\mu(s) \alpha_{1}} \frac{e_{-\alpha}(s, \tau)}{e_{\alpha 1}(\tau, s)} \Delta s \leq l, \quad t \geq \tau,
$$

hold. Then Eq.(3.1) is exponentially stable.

Proof. The equation $g^{\Delta}-D g-f=0$ is exponentially stable, by Theorem 2.1, and any solution $g(t)=g\left(t, \tau, g_{\tau}\right)$ with initial value $g_{\tau}$, satisfies

$$
\|g(t)\| \leq\left(\gamma(\tau)\left\|g_{\tau}\right\|+\beta(\tau)\right) e_{-\alpha}(t, \tau) \quad \forall t \geq \tau, t \in \mathbb{T}
$$

Set

This gives

$$
\gamma(\tau, r)=\gamma(\tau) r+\beta(\tau)
$$

$$
\|g(t)\| \leq \gamma_{1}\left(\tau,\left\|g_{\tau}\right\|\right) e_{-\alpha}(t, \tau) \forall t \geq \tau, t \in \mathbb{T}
$$


Let $x(t)$ be a solution of Eq.(3.1) with initial value $X(\tau)=\left(x_{\tau}^{0}, x_{\tau}^{1}\right)$. Then $g(t)=x^{\Delta}(t)+$ $z(t) x(t)$ is a solution of Eq. (3.3) with initial value $g(\tau)=x_{\tau}^{1}+z(\tau) x_{\tau}^{0}$. The solution $x(t)$ is given by

Hence

$$
x(t)=e_{-z}(t, \tau) x_{\tau}^{0}+\int_{\tau}^{t} e_{-z}(t, \sigma(s)) g(s) \Delta s
$$

$$
\begin{gathered}
\|x(t)\| \leq \gamma(\tau)\|X(\tau)\| e_{-\alpha 1}(t, \tau) \\
\quad+\int_{\tau}^{t} \gamma(\sigma(s)) e_{-\alpha}(t, \sigma(s)) \gamma_{1}\left(\tau,\left\|g_{\tau}\right\|\right) e_{-\alpha}(s, \tau) \Delta s \\
\leq\left[\gamma(\tau)\|X(\tau)\|+\gamma_{2}(\tau,\|X(\tau)\|) l\right] e_{-\alpha}(t, \tau), \forall t \in \mathbb{T}, t \geq \tau,
\end{gathered}
$$

where $\gamma_{2}(\tau, r)=\gamma(\tau) \backslash \max (\|z(\tau)\|, 1) r+\beta(\tau)$.Therefore, Eq. (3.1) is exponentially stable.

Theorem 3.5. Assume that the following conditions

i. $\quad$ There exist constants $\alpha>0, \alpha_{1}>0$ and $\gamma \geq 1$, such

that $\left\{e_{-} D(t, \tau): t \geq \tau\right\}$ ( resp. $\left\{e_{-Z}(t, \tau): t \geq \tau\right\}$ ) is stable, with type $\alpha$ and $\gamma$ ( resp. with type $\alpha_{1}$ and $\left.\gamma\right)$

ii. There are constants $\beta>0$ and $l>0$, independent on $\tau$, such that

$$
\int_{\tau}^{t} \frac{\|f(s)\|}{1-\mu(s) \alpha} e_{-z}(\tau, s) \Delta s \leq \beta, \quad t \geq \tau
$$

and

$$
\int_{\tau}^{t} \frac{\gamma(\sigma(s))}{1-\mu(s) \alpha_{1}} \frac{e_{-\alpha}(s, \tau)}{e_{\alpha 1}(\tau, s)} \Delta s \leq l, \quad t \geq \tau, \tau \in \mathbb{T}
$$

are satisfied. Then Eq.(3.1) is uniformly exponentially stable.

Proof.The proof is similar to the proof of Theorem 3.4 and will be omitted.

Theorem 3.6. Let $h$ and $h_{1}$ be positive bounded functions on $\mathbb{T}$. Assume that the following conditions

i. There exist $\gamma \in C_{r d}\left(\mathbb{T}, \mathbb{R}^{\geq 1}\right)$ such that $\left\{e_{D}(t, \tau): t \geq \tau\right\}$ is $h$-staple with type $\gamma(\tau)$ and $\left\{e_{-z}(t, \tau): t \geq \tau\right\}$ is $h_{1}$-staple with same type $\gamma(\tau)$.

ii. There are constants $\beta>0$ and $l>0$, dependent on $\tau$, such that

$$
\int_{\tau}^{t} \frac{\|f(s)\| \gamma(\sigma(s))}{h(\sigma(s)) h(\tau)^{-1}} \Delta s \leq \beta, \quad t \geq \tau \text { and } \int_{\tau}^{t} \frac{\gamma(\sigma(s))}{h_{1}(\sigma(s)) h_{1}(\tau)^{-1}} h(\sigma(s)) h(\tau)^{-1} \Delta s \leq l
$$

are satisfied. Then Eq.(3.1) ish-stable. 
Proof. The equation $g^{\Delta}-D g-f=0$ is $h$ - stable, by Theorem 2.3, and any solution $g(t)=g\left(t, \tau, g_{\tau}\right)$ with initial value $g_{\tau}$, satisfies

$$
\|g(t)\| \leq\left(\gamma(\tau)\left\|g_{\tau}\right\|+\beta\right) h(t) h(\tau)^{-1} \forall t \geq \tau, t \in \mathbb{T}
$$

Set

This gives

$$
\gamma_{1}(\tau, r)=\gamma(\tau) r+\beta
$$

$$
\|g(t)\| \leq \gamma_{1}\left(\tau,\left\|g_{\tau}\right\|\right) h(t) h(\tau)^{-1} \forall t \geq \tau, t \in \mathbb{T} .
$$

Let $x(t)$ be a solution of Eq.(3.1) with initial value $X(\tau)=\left(x_{\tau}^{0}, x_{\tau}^{1}\right)$. Then $g(t)=x^{\Delta}(t)+$ $z(t) x(t)$ is a solution of Eq. (3.3) with initial value $g(\tau)=x_{\tau}^{1}+z(\tau) x_{\tau}^{0}$. The solution $x(t)$ is given by

Hence

$$
x(t)=e_{-z}(t, \tau) x_{\tau}^{0}+\int_{\tau}^{t} e_{-z}(t, \sigma(s)) g(s) \Delta s .
$$

$$
\begin{aligned}
& \|x(t)\| \leq \gamma(\tau)\|X(\tau)\| h(t) h(\tau)^{-1} \\
& \quad+\gamma_{1}\left(\tau,\left\|g_{\tau}\right\|\right) h_{1}(t) \int_{\tau}^{t} \frac{\gamma(\sigma(s)) h(s)}{h_{1}(\sigma(s))} h(\tau)^{-1} \\
& \quad \leq\left[\gamma(\tau)\|X(\tau)\|+\gamma_{1}\left(\tau,\left\|g_{\tau}\right\|\right) l\right] h(t) h(\tau)^{-1} \\
& \leq\left[\gamma(\tau)\|X(\tau)\|+\gamma_{2}(\tau,\|X(\tau)\|) l\right] h(t) h(\tau)^{-1}, \forall t \in \mathbb{T}, t \geq \tau,
\end{aligned}
$$

where $\gamma_{2}(\tau, r)=\gamma(\tau) \backslash \max (\|z(\tau)\|, 1) r+\beta(\tau)$. Therefore, Eq. (3.1) is $h$ - stable.

Theorem 3.7. Let $h$ and $h_{1}$ be positive bounded functions on $\mathbb{T}$. Assume that the following conditions

i. There exist $\gamma \geq 1$ such that $\left\{e_{D}(t, \tau): t \geq \tau\right\}$ is uniformly $h$-staple with type $\gamma$ and $\left\{e_{-z}(t, \tau): t \geq \tau\right\}$ is uniformly $h_{1}$-staple with same type $\gamma$.

ii. There are constants $\beta>0$ and $l>0$, independent on $\tau$, such that

$$
\int_{\tau}^{t} \frac{\|f(s)\|}{h(\sigma(s)) h(\tau)^{-1}} \Delta s \leq \beta, t \geq \tau \text { and } \int_{\tau}^{t} \frac{h(\sigma(s)) h(\tau)^{-1}}{h_{1}(\sigma(s)) h_{1}(\tau)^{-1}} \Delta s \leq l
$$

are satisfied. Then Eq.(3.1) is uniformly $h$ - stable.

Proof.The proof is similar to the proof of Theorem 3.4 and will be omitted.

Theorem 3.6 and 3.7 yield Theorem 3.4 and 3.5 respectively, by putting $h(t) h(\tau)^{-1}=e_{-\alpha}(t, \tau)$

\section{Illustrative examples}


The following examples show the applicability of the main results. In all examples $\mathbb{X}$ denotes a Banach space endowed with a norm \| $\|$ and $I$ denotes the identity operator on $\mathbb{X}$.

\section{Example 4.1.}

Consider the following dynamic equation

$$
\left.x^{\Delta \Delta}(t)+A(t) x^{\Delta}(t)+R(t) x(t)=0, t \in \mathbb{T}=\backslash \mathbb{R}^{\geq 0}\right\}
$$

where $A(t)=2 t I$ and $R(t)=\left(1+t^{\wedge} 2\right) I$. The corresponding Riccati equation is

$$
z^{\Delta}(t)-\left(z^{\sigma}(t)-A(t)\right) z(t)=R(t), t \in \mathbb{T} .
$$

One can see that $z(t)=t I$ is a solution of Eq.(4.2). We have,

$$
D(t)=z^{\sigma}(t)-A(t)=-t I=-z(t), t \in \mathbb{T} .
$$

We have

$$
e_{D}(t, \tau)=e^{-\frac{t^{2}-\tau^{2}}{2}} I
$$

see for instance [1], [13], and consequently,

$$
\left\|e_{D}(t, \tau)\right\|=\left\|e_{-z}(t, \tau)\right\|=e^{-\frac{t^{2}-\tau^{2}}{2}}
$$

Since $\left\|e_{D}(t, \tau)\right\|,\left\|e_{-z}(t, \tau)\right\|$ and $\int_{\tau}^{t}\left\|e_{-z}(t, \sigma(s))\right\| d s$ sare uniformly bounded with respect to $\tau$, then by Theorem 3.2, Eq.(4.1) is stable.

In this example, if we take $\mathbb{T}$ is any closed bounded interval, then $\|z(t)\|$ is bounded on this interval. Consequently, by Theorem 3.3, Eq.(4.1) is uniformly stable.

Example 4.2. Consider the following dynamic equation

$$
x^{\Delta \Delta}(t)+A(t) x^{\Delta}(t)+R(t) x(t)=f(t), t \in \mathbb{T}=\mathbb{R}^{+},
$$

Where $A(t), R(t)$ and $f(t)$ defined by

$$
A(t)=2 m I, R(t)=m^{2} I \text { and } f(t)=e^{-2 m t} a,
$$

where $m>0, a \in \mathbb{X}$. The corresponding Riccati equation is

$$
z^{\Delta}(t)-\left(z^{\sigma}(t)-A(t)\right) z(t)=R(t), t \in \mathbb{T} .
$$

One can see that $z(t)=m I$ is a solution of Eq.(4.4). We note that

$$
D(t)=z^{\sigma}(t)-A(t)=-m I=-z(t), t \in \mathbb{T},
$$

and $\left\|e_{D}(t, \tau)\right\|=\left\|e_{-z}(t, \tau)\right\|=e_{-m}(t, \tau)$. Choosing $\alpha=\alpha_{1}=m$ and $\gamma=1$ in Theorem 3.5 , simple calculations show that 


$$
\int_{\tau}^{t} \frac{\|f(s)\| \gamma(\sigma(s))}{1-\mu(s) \alpha} e_{-\alpha}(\tau, s) d s=\int_{\tau}^{t} e^{-2 m s} e^{-m(\tau-s)} d s<\frac{1}{m}, \quad t, \tau \in \mathbb{T}, t \geq \tau,
$$

and

$$
\int_{\tau}^{t} \frac{\gamma(\sigma(s))}{1-\mu(s) \alpha_{1}} \frac{e_{-\alpha}(s, \tau)}{e_{-\alpha_{1}}(\tau, s)} d s=\int_{\tau}^{t} e^{-2 m(s-\tau)} d s<\frac{1}{2 m}, \quad t, \tau \in \mathbb{T}, t \geq \tau,
$$

Therefore, Eq.(4.3) is uniformly exponentially stable.

Example 4.3. Consider the following dynamic equation

$$
x^{\Delta}(t)=A(t) x(t), t \in \mathbb{T}=\mathbb{R},
$$

where $A(t)=-m I$ such that $m>0$. We note that $\left\|e_{A}(t, \tau)\right\|=e_{-m}(t, \tau)$. Choosing $\alpha=m$ and $\gamma=1$ in Theorem 3.3 conditions will be realized. Therefore, Eq.(4.5) is uniformly exponentially stable.

\section{References}

[1] M. A. Al-Qubaty, On The Theory of Dynamic Equations On Time Scales, Ph. D. Thesis, Ain Shams University, (2012).

[2] P. J. Antsaklis and A. N. Michel, A Linear Systems Primer, Birkhauäser Boston, (2007).

[3] B. Aulbach and S. Hilger, A Unified Approach to Continuous and Discrete Dynamics, Qualitative Theory of Differential Equations (Szeged, 1988), Colloq. Math. Soc. Janos Bolyai. North Holland, Amesterdam, Besel, (2001).

[4] M. Bohner and A. Peterson, Dynamic Equations On Time Scales: An Introductionwith Applications, Birkhäuser, Boston. Basel. Berlin, (2001).

[5] M. Bohner and A. Peterson, Advanced in Dynamic Equations on Time Scales, Birkhäuser, Boston. Basel. Berlin, (2003).

[6] S. K. Choi and N. J. koo, Sability of Linear Dynamic Equations on Time Scales, Discrete and Continuous Dynamical Systems , (2009), 161-170.

[7] S. K. Choi, N. J. koo and D. M. Im, h-Sability for Linear Dynamic Equations on Time Scales, J. Math. Anal.Appl., 324 (2006), 707-720.

[8] J. J. Dacunha, Stability for Time Varying Linear Dynamic Systems on Time Scales, Journal of Computational and Applied Mathematics 176 (2005). Stability of Abstract Linear Dynamic Equations 13

[9] A. Drozdowicz and J. Popenda, Asymptotic Behavior of Solutions of Difference Equations of Second Order, Journal of Computational and Applied Mathematics,47 (1993), 141-149. 
[10] L. H. Duc, A. Ichlmann, S. Siegmund and P. Taraba, On Stability of Linear TimeVarying Second-Order Differential Equations, American Mathematical Society; 64 (1) (2006), 137-151.

[11] S. N. Elaydi, An Introduction to Difference Equations, Springer-Verlag, New York, (1996).

[12] A. E. Hamza and K. M. Oraby, Stability of Abstract Dynamic Equations on Time Scales, Advances in Difference Equations, (2012), 2012:143.

[13] A. E. Hamza and M. A. Al.Qubaty, On The Exponential Operator Functions on Time Scales, Advances in Dynamical Systems and Applications,7 (1) (2012), 57-80.

[14] S. Hilger, Ein Mabkettenkolkül mit Anwendung auf Zentrumsmanningfaltigkeiten, Ph. D. Thesis, Universität Würzburg, (1988).

[15] S. Hilger, Analysis on Measure Chains- a Unified Approach to Continuous and Discrete Calculus, Results Math. 18 (1990) 18-56.

[16] X. Liao, L. Wang and P.YU, Stability of Dynamical Systems, Elsevier, (2007).

[17] Nasser, B. B., Boukerriona, K. and Hammami, M. A., On Stability and Stbilization of Perturbed Time Scale Systems with Gronwall Inequalities, Journal of Mathematical Physics, Analysis, Geometry, 11 (2015), 207-253.

[18] W. J. Rugh, Linear System Theory, Second Edition, Prentice-Hall, Englewood Cliffs, (1996). 


\section{الملخص بللغفه العربيه}

اسم الطالب: دينا احمد محمد ابراهيم

عنوان البحث: انواع عديده من الاستقراريه لمعادلات الديناميكيه من الارجه الأولي و الثانيه علي مقاييس الزمن قمنا في هذه الرساله بأستخدام داله ليابونوف مناسبه و وضعنا الثروط الكافيه للاستقر ارية المنتظمه و الاستقر اريه الأسية و الاستقر اريه الأسية المنتظمه و الاستقر اريه من النوع (h) و الاستقر اريه من النوع (h) المنتظمه لمعادله ديناميكيه من الدرجه الاولي علي مقاييس الزمن T ذات الاسية الصيغه

و معادله ديناميكيه من الدرجه الثانيه علي مقاييس الزمن T ذات الصيغه

حيث ان (X) نهايه البحث نم عمل تطبيقات للنظريه الني حصلنا عليها.

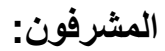

أ.د. علاء الدين حمزه سيد

أستاذ الرياضيات البحته ــ كليه العلوم- جامعه القاهره أ.د. جمال علي فؤاد اسماعيل

أستاذ الرياضيات البحته ــ كليه البنات- جامعه عين شمس 
